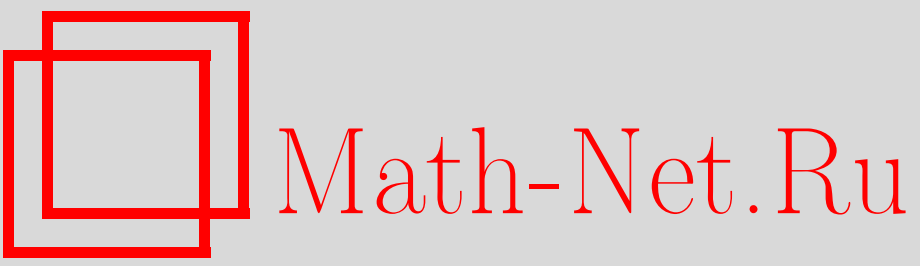

С. М. Натанзон, Вещественные оснащения комплексных алгебраических кривых и группы Кокстера, УМH, 1996, том 51, выпуск 6, 215-216

DOI: https://doi.org/10.4213/rm1034

Использование Общероссийского математического портала Math-Net.Ru подразумевает, что вы прочитали и согласны с пользовательским соглашением

http://www.mathnet.ru/rus/agreement

Параметры загрузки:

IP: 18.234 .197 .8

26 апреля 2023 г., 13:58:58 


\title{
ВЕЩЕСТВЕННЫЕ ОСНАЩЕНИЯ КОМПЛЕКСНЫХ АЛГЕБРАИЧЕСКИХ КРИВЫХ И ГРУППЫ КОКСТЕРА
}

\author{
C. M. НАТАНЗОН
}

1. Пусть $P$ - комплексная алгебраическая кривая, т.е. компактная риманова поверхность рода $g(P)$. Антиголоморфная инволюция $\tau: P \rightarrow P$ называется вещественной формой кривой $P[1]$. Она определяет на $P$ структуру вещественной алгебраической кривой с вещественными точками $P^{\tau}=\{p \in P \mid \tau p=p\}$ (см. [2])

Вещественная форма $\tau$ называется ориентируемой, если $P /\langle\tau\rangle$ - ориентируемая поверхность. В этом случае вещественные точки $P^{\tau}$ делят $P$ на две поверхности. Ориентируемыми являются, например, $M$-формы, играющие особую роль в теории вещественных кривых [3].

Вещественным ориентируемым оснащением назовем пару $(P, W)$, где $W$ - группа, порожденная ориентируемыми вешественными формами. Пусть $P^{W}$-множество всех вешественных точек всех ориентируемых вешественных форм из $W$. Рассмотрим замыкание $C$ компоненты связности дополнения $P \backslash P^{W}$. Базисом оснащения назовем множество $S=\left\{\tau_{1}, \ldots, \tau_{n}\right\}$ всех вещественных форм $\tau_{i} \in W$ таких, что $P^{\tau_{i}} \cap C \neq \varnothing$.

ЛЕмма 1. Любая ориентируемая вещественная форма $\tau \in W$ сопряжена в группе $W$ одной из форм $\tau_{i} \in S$. Мнохсество $S$ порождает $W$.

ДоказАТЕЛЬСтво. Рассмотрим группу $\widetilde{W}$, порожденную $S$. Множество $\widetilde{P}=\bigcup_{\widetilde{w} \in \widetilde{W}} C \subset P$ компактно и не имеет границ. Следовательно, $\widetilde{P}=P$. Поэтому существует $\widetilde{w} \in \widetilde{W}$ такой, что $\widetilde{w} C \cap P^{\tau} \neq \varnothing$ и, следовательно, $\widetilde{w} P^{\tau_{i}} \cap P^{\tau} \neq \varnothing$ для некоторого $\tau_{i} \in S$. Но тогда $\tau=\widetilde{w} \tau_{i} \widetilde{w}^{-1}$.

Обозначим через $W_{i}$ множество таких $w \in W$, что $C$ и $w(C)$ лежат в одной компоненте связности множества $P \backslash P^{\tau_{i}}$.

ЛЕмма 2. Если $w \in W_{i} u w \tau_{j} \notin W_{i}, m o w \tau_{j}=\tau_{i} w$.

ДоКАЗАТЕЛЬСТво. По условию $w \tau_{j}(C)$ и $w(C)$ лежат в разных компонентах множества $P \backslash P^{\tau_{i}}$. Следовательно, $\tau_{j}(C)$ и $C$ лежат в разных компонентах множества $P \backslash P^{w^{-1}} \tau_{i} w$. Но они имеют общую границу $P^{\tau_{j}}$ и, следовательно, $P^{\tau_{j}} \subset P^{w^{-1}} \tau_{i} w$, откуда $\tau_{j}=w^{-1} \tau_{i} w$.

Обозначим через $l(w)$ наименшшее число $l=l(w)$ такое, что $w=\tau_{i_{1}} \cdots \tau_{i_{l}}$.

Теорема 1. 1) Пара $(W, S)$ является системой Кокстера, т.е. S порождает W определяющими соотношениями $\tau_{i}^{2}=1,\left(\tau_{i} \tau_{j}\right)^{m_{i j}}=1$.

2) $C-$ фундаментальная область группь $W$.

ДоказАтельство. Согласно $[4, \mathrm{IV}, \S 1$, п. 7$]$ из очевидных соотношений $1 \in W_{i}, W_{i} \cap \tau_{i} W_{i}$ $=\varnothing$ и утверждения леммы 2 следует, что $(W, S)$ является системой Кокстера и

$$
W_{i}=\left\{w \in W \mid l\left(\tau_{i} w\right)>l(w)\right\} .
$$

Следовательно, если $w C=C$, то

$$
w \in \bigcap_{i=1}^{n} W_{i}=\left\{w \in W \mid l\left(\tau_{i} w\right)>l(w) \text { при } i=1, \ldots, n\right\}=1 .
$$

СлЕДСТВИЕ 1. Пусть $(P, W)$ - вещественное ориентируемое оснащение. Тогда

1) все неподвижные точки всех автоморфизмов $w \in W$ принадлежат $P^{W}$;

2) всякая вещественная форма с вещественными точками $\tau \in W$ сопряжена одной из форм $\tau_{i} \in S$.

Работа выполнена при финансовой поддержке Российского фонда фундаментальных исследований (грант № 95-01-01122a). 
ДокАЗАТЕльСтво. Утверждение 1) следует из фундаментальности области $C$. Утверждение 2) следует из 1) и леммы 1.

2. Полная классификация пар Коксткра $(W, S)$ хорошо известна $[4, \mathrm{VI}, \S 4$, п. 1]. Они полностью описываются своим графом Кокстера (диаграммой Дынкина), полный список которых также хорошо известен. Это позволяет перейти к полной топологической классификации вещественных ориентируемых оснащений.

Два таких оснащения $\left(P_{1}, W_{1}\right)$ и $\left(P_{2}, W_{2}\right)$ назовем топологически әквивалентными, если существует гомеоморфизм $\varphi: P_{1} \rightarrow P_{2}$ такой, что $W_{2}=\varphi W_{1} \varphi^{-1}$.

Фундаментальная область $C$ гомеоморфна поверхности рода $g(P / W)$ с $k(P / W)$ дырами. Граница каждой дыры $a^{\alpha} \subset C(\alpha=1, \ldots, k)$ состоит из упорядоченных согласно ориентации $P / W$ отрезков $l_{\beta}^{\alpha}$, где $\beta \in Z_{m_{\alpha}}$ и $l_{\beta}^{\alpha} \cap l_{\beta+1}^{\alpha} \neq \varnothing$. При этом $l_{\beta}^{\alpha} \in P^{\tau_{\beta}^{\alpha}}$, где $\tau_{\beta}^{\alpha} \in S$. Набор $\left\{\tau_{\beta}^{\alpha}\right\}$ назовем $C$-типом оснашения $(P, W)$. Он определен с точностью до замен $\left\{\tau_{-\beta}^{\alpha}\right\}$ и $\tau_{\beta+\gamma_{\alpha}}^{\alpha}$, где $\gamma_{\alpha} \in Z_{m_{\alpha}}$. При замене $C C$-тип переходит в сопряженньй.

Теорема 2. 1) Вещественные ориентируемые оснащения $\left(P_{1}, W_{1}\right)$ и $\left(P_{2}, W_{2}\right)$ топологически әквивалентны, если и только если $g\left(P_{1} / W_{1}\right)=\left(P_{2} / W_{2}\right)$ и существует изоморфизм $\psi: W_{1} \rightarrow W_{2}$, переводящий $C_{1}$-тип оснащения $\left(P_{1}, W_{1}\right)$ в $C_{2}$-тип оснащения $\left(P_{2}, W_{2}\right)$ для некоторьх фундаментальных областей $C_{i} \subset P_{i}$.

2) Пусть $(\widetilde{W}, \widetilde{S})$ - произвольная кокстеровская система и $\widetilde{\tau}_{\beta}^{\alpha} \in \widetilde{S}-$ набор образующих $\widetilde{W}$, где $\alpha=1, \ldots, k, \beta \in Z_{m_{\alpha}} u \tau_{\beta+1}^{\alpha} \neq \tau_{\beta}^{\alpha}$. Тогда для любого $g \geqslant 0$ существует вещественное ориентируемое оснащение $(P, W)$ и изоморфизм $\varphi: W \rightarrow \widetilde{W}$ такие, что $g(P / W)=g$ и $\tau_{\beta}^{\alpha}=\varphi\left(\widetilde{\tau}_{\beta}^{\alpha}\right)-C$-тип $(P, W)$ для некоторой фундаментальной области $C$ әруппь $W$.

ДокАЗАТЕЛЬСтво в случае $g\left(P_{i} / W_{i}\right)=0$ следует из представления оснащения $(P, W)$ в виде $(\Lambda / \Gamma, \widetilde{\Gamma} / \Gamma)$, где $\widetilde{\Gamma}-N E C$-группа и $\Gamma \triangleleft \widetilde{\Gamma}$ - фуксова группа на плоскости Лобачевского [5]. Общий случай легко следует из случая $\left(g / P_{i} / W_{i}\right)=0$ с помощью разрезов по контурам, окружающим дыры.

Из следствия 1 и теоремы Римана-Гурвица сразу следует

Теорема 3. Пусть $(P, W)$ - вещественное ориентируемое оснащение $C$-типа $\tau_{\beta}^{\alpha}$ $\left(\alpha=1, \ldots, k, \beta \in Z_{m_{\alpha}}\right)$. Пусть $|W|-$ порядок группь $W$ и $n_{\beta}^{\alpha}-$ порядок автоморфизма $\tau_{\beta}^{\alpha} \cdot \tau_{\beta+1}^{\alpha}$. Тогда $g(P)-1=\frac{|W|}{2}\left(2 g(P / W)+k(P / W)-1+\frac{1}{2} \sum_{\alpha \beta}\left(1-\frac{1}{n_{\beta}^{\alpha}}\right)\right)$.

СледСтвиЕ 2 [6]. Число попарно несопряженных ориентируемых вещественных форм у кривой рода $g$ не превосходит $(g(P)-1) / 2^{n-3}$.

СлЕдСтвиЕ 3. Всякий имеющий неподвижные точки голоморфный автоморфизм $\alpha \in W$ порядка больие 5 порождает нормальный делитель группь $W$.

ДоказАтельство. Пусть $C$ - фундаментальная область, содержащая неподвижные точки $\alpha$, и $S=\left\{\tau_{1}, \ldots, \tau_{n}\right\}$ - отвечающий ей базис. Согласно следствию $1 \alpha=\left(\tau_{i} \cdot \tau_{j}\right)^{m}$. Но граф Кокстера системы Кокстера $(W, S)$ распадается на связные графы, среди которых ребра порядка более 5 имеют лишш графы из двух вершин [4, VI, $\S 4]$. Таким образом, $\alpha$ коммутирует со всем множеством $S \backslash\left(\tau_{i} \cup \tau_{j}\right)$.

\section{СПИСОК ЛИТЕРАТУРЫ}

[1] Натанзон C. M. // Труды MMO. 1988. T. 51. C. 3-53. [2] Alling N. L., Greenleaf N. // Lecture Notes in Math. 1971. V. 219. [3] Natanzon S. M. // Selecta Math. Soviet. 1981. V. 1. P. 81-99. [4] Бурбаки Н. Группы и алгебры Ли. М.: Мир, 1972. [5] Bujalance E., Etayo J. J., Gamboa J. M., Gromadzki G. // Lecture Notes in Math. 1990. V. 1439. [6] Bujalance E., Gromadzki G., Singerman D. // Proc. Amer. Math. Soc. 1994. V. 120. № 2. P. 507-513. 